\title{
Research Progress of Th17/Treg Cells and Their Transcription Factors in Autoimmune Diseases
}

\author{
Zhenjiang Hou ${ }^{1, ~ *}$, Zhaoxin Mu ${ }^{1}$, Cuicui Wang ${ }^{2}$ \\ ${ }^{1}$ Institute of Thyroid Diseases Affiliated to Cangzhou Medical College, Cangzhou Thyroid Disease Engineering Technology Research Center, \\ Cangzhou, China \\ ${ }^{2}$ Department of Medical Technology Laboratory, Cangzhou Medical College, Cangzhou, China
}

Email address:

houzhenjiang@sina.com (Zhenjiang Hou)

${ }^{*}$ Corresponding author

To cite this article:

Zhenjiang Hou, Zhaoxin Mu, Cuicui Wang. Research Progress of Th17/Treg Cells and Their Transcription Factors in Autoimmune Diseases. American Journal of Clinical and Experimental Medicine. Vol. 7, No. 4, 2019, pp. 83-92. doi: 10.11648/j.ajcem.20190704.12

Received: July 9, 2019; Accepted: September 24, 2019; Published: September 27, 2019

\begin{abstract}
Helper T cell 17 (Th17) being a new cell subset of CD4+T in the body, which is different from Th1 and Th2 cells, have independent mechanisms of differentiation and developmental regulation. Th17 cells mainly secrete various cytokines such as IL-17, IL-6 and TNF- $\alpha$, which induce inflammation. Treg cells are T cells with high expression of CD25 differentiated by $\mathrm{T}$ cells under the action of certain cytokines, namely CD4+CD25+(hi) Foxp3+T cells, which can regulate the immune response mediated by effector cells and are an important line of defense for human autoimmune. Therefore, Treg cells play an important role in maintaining immune tolerance of the body. As an important subset of T cells, Treg cells have an inhibitory effect on inflammation. Its working principle is to selectively inhibit autoreactive $\mathrm{T}$ cells and effector $\mathrm{T}$ cells so as to maintain the body's immune balance, and normal quantity and function help the immune system to its antigen stimulation, establishing a good state of tolerance. The expression of Treg cells increased, can avoid the occurrence of autoimmune diseases. Treg cells inhibit the differentiation of Th17 cells by down-regulating the expression of IL-23 and IL-17 or by its specific transcription factor Foxp3; similarly, inhibition of Th17 cell production can promote the development of Treg cells. Both Th17 and Treg cells are functionally inhibited. Th17 cells promote inflammatory reaction, and Treg cells suppress immune reaction. Numerous cytokines are involved in regulation. For example, IL-6 and IL-21 can inhibit Foxp3 and promote the expression of ROR $\gamma t$, thereby inhibiting Treg cells and inducing the differentiation of Th17 cells. In the absence of IL- 6 and other pro-inflammatory factors, TGF- $\beta$ enhances the inhibitory effect of Foxp3 on ROR $\gamma \mathrm{t}$ and promotes the growth and development of Treg cells. The anti-inflammatory factor IL-10 also induces Treg cells to inhibit the reaction of Th17 cells. The effects of Treg and Th17 cells are normally in a dynamic equilibrium. Once the balance is imbalanced, autoimmune diseases will occur. This article reviews the differentiation, function and research progress of Th17/Treg cells in autoimmune diseases.
\end{abstract}

Keywords: Helper T 17 Cell, Regulatory T Cell, Retinoidrelated Orphan Receptors - $\gamma$ t, Forkhead/Winged, Helix Transcription Factor 3, Autoimmune Diseases

\section{Introduction}

Th17 cells are a new type of effector CD4+T cells subset that is different from Th1 and Th2 cells. Characterized by specific secretion of high levels of interleukin 17 (IL-17), it is involved in the occurrence and development of inflammatory responses and autoimmune diseases [1]. As a kind of immunosuppressive $\mathrm{T}$ cell, regulatory $\mathrm{T}$ cell (Treg) plays an antagonistic role with Thl7 cells and plays a regulatory role in immune inflammatory response, which is a key factor to maintain peripheral immune tolerance [2]. Loss or dysfunction of Treg cell can lead to autoimmune damage of multiple organs or even death. The recent research found that Retinoidrelated Orphan Receptors (ROR- $\gamma \mathrm{t}$ ) and Forkhead/winged helix transcription factor 3 (Foxp3) were specific transcription factors of Th17 and Treg cells, respectively and played a very important role in immunosuppression $[3,4]$. The TGF- $\beta$ signaling pathway plays an important role in the differentiation of Th17 and 
Treg cells. The balance of th17 and Treg cells plays an important role in autoimmune tolerance and immune homeostasis, and has become the research direction of the occurrence, development and prognosis of immune-related diseases. The imbalance between Th17 and Treg cells is closely connected with a variety of immune diseases, such as autoimmune diseases [3]. Th17 cell and its secreted IL-17 expression and dysfunction are closely related to the development of autoimmune diseases. The increased expression of Treg cells avoids the occurrence of autoimmune diseases.

\section{Differentiation and Function of Th17/Treg Cells}

\subsection{Differentiation and Function of Th17 Cells}

Th17 cell are a new type of effector CD4+T cells subset which is different from Th1 and Th2 cells, characterized by specific secretion of high levels of interleukin 17(il-17) [5]. The differentiation of Th17 cells mainly includes three stages:(1) Induced differentiation: low concentration of transforming growth factor- $\beta$ (TGF- $\beta$ ) and interleukin 6 , IL-6, enable CD4+ $\mathrm{T}$ cells to differentiate into Th17 cells by activation the ROR $\gamma$ t transcription factor [6]. ROR $\gamma t$ is a Th17 cell-specific transcriptional regulator that requires the involvement of ROR $\gamma \mathrm{t}$ regardless of the differentiation of Th17 cells induced by cytokines in vitro or the Th17 cell-mediated inflammatory response in vivo. In addition, transcription factors such as ROR $\alpha$, AHR, AP-1, and Batf are also involved in the differentiation of Th17 cells; (2) In vivo expansion: CD4+T cells begin to secrete IL-21 after differentiation into Th17 cells, further promoting their differentiation and proliferation. TGF- $\beta$ plays a synergistic role in the amplification of Th17; (3) Maintenance function: dendritic cells (DCs) and other antigen-presenting cells (APC) produce IL-23, which plays a major role in maintaining the survival and function of Th17 cells. IL-6, IL-21 and IL-23 can also activate the downstream signaling pathway, induce the expression of IL-23 receptor on the surface of Th17 cells, and further maintain the function of Th17 cells [7]. The simultaneous expression of TGF- $\beta$ and IL- 6 can induce the high expression of ROR $\gamma \mathrm{t}$, which differentiates naive CD4+T cells into Th17 and stimulates the secretion of IL-17, indicating that the pro-inflammatory cytokines IL-6 and TGF- $\beta$ secreted by immune cells are involve in the differentiation of Th17 cells [8]. Researches have shown that differentiated mature Th17 cells secrete a variety of cytokines such as IL-17A, IL-17F, IL-21, IL-22, which are involve in the development of inflammatory reactions, infections and autoimmune diseases.

\subsection{Differentiation and Function of Treg Cells}

Regulatory $\mathrm{T}$ cells (Tregs) are a subset of CD4+ $\mathrm{T}$ lymphocytes, which are classified into naturally occurring $\mathrm{T}$ cells (nTreg) and induced $\mathrm{T}$ cells (inducedregulatory $\mathrm{T}$ celli
Treg) according to the source of Treg cells. The former migrated to the periphery after thymus maturation, accounting for $5 \%$ to $10 \%$ of peripheral blood CD4+T cells. Immune tolerance is mainly induced by immediate contact between cells, which is closely related to autoantigen ${ }^{[5]}$; The latter is expressed by mature $\mathrm{T}$ cells or initial $\mathrm{T}$ cells in peripheral lymphocyte tissues through antigenic action and induced differentiation through TGF- beta expression of forhead/winged helix transcription factor (Foxp3), which can also be transformed by nTreg under certain conditions $[9,10]$. The phenotype of Treg cells is CD4+CD25+Foxp3+, which exerts immunosuppressive effects in immune tolerance and autoimmune protection by secreting cytokines such as TGF- $\beta$, IL-10 and IL-35 [11]. Treg cells play an important role in the occurrence and development of autoimmune diseases through the interaction of many cytokines. The abnormal number and lack of function of Treg cells [12] are involved in the development of various autoimmune diseases.

\section{Th17 / Treg Cells Imbalance and Autoimmune Diseases}

\subsection{Th17 / Treg Cells and Rheumatoid Arthritis}

\subsubsection{Th17 Cells and Rheumatoid Arthritis}

Rheumatoid arthritis (RA) is a common chronic inflammatory autoimmune disease, which is mainly manifested by chronic progressive and invasive inflammation of the synovium of the joint. The pathogenesis of RA may be bone and cartilage destruction by inflammatory cells, eventually leading to severe disability and increased mortality. Animal experiments and clinical researches have confirmed that Th17 cells are in an unbalanced state during the occurrence and development of RA. Animal experiment-collagen-induced arthritis (CIA) found that IL-17 in knee joint organized expression of collagen type II immune mice, which can promote collagen-induced arthritis and aggravate joint destruction [13]. Th1 cells in mice that defects IL-23 responded normally, while Th17 cells developed defects couldn't significantly inhibited the development of CIA, which indicated that Th17 cells, rather than Th1 cells, played a key role in the development, progression and pathogenesis of RA [14]. It was confirmed that Th17 cells played an important role in the development of the CIA mouse model. The absence of IL-17 or the treatment of CIA with IL-17 monoclonal antibody can improve joint inflammation in mice, reducing the degree of joint damage, bone erosion and cartilage destruction. Wang et al [15] found that a group of Th17-like Tregs in the synovial fluid and peripheral blood of RA patients, actually was $\mathrm{CD}+4 \mathrm{CD}+25 \mathrm{CD}+161 \mathrm{Treg}$, which was named for its similar properties to the pro-inflammatory subpopulation Th17. It is suggested that Treg not only has anti-inflammatory effects, but also can transform into pro-inflammatory Th17 cells and accelerate the progression of arthritis when the body's immune disorder is unbalanced. Lee et al [16] reported that the number of Th17 cells in RA patients was significantly 
higher than that in the control group, and the amount of synovial fluid was related to the serum IL-17A level and the severity of joint damage. Blocking IL-17A and TNF- $\alpha$ has a synergistic effect on inhibiting IL-6 production and collagen degradation in RA synovial fluid and synovial fluid [17]. Therefore, Th17 cells can be used as a new target for the treatment of RA.

\subsubsection{Treg Cells and Rheumatoid Arthritis}

The main function of Treg cells is to mediate immune tolerance, inhibit autoimmune responses, and thereby slow the development of arthritis in mice. Numerous cytokines can affect the inhibitory function of Treg cells. For example, IL-21 produced by Th17 cells makes effector T cells resistant to the inhibitory function of Treg cells [18]. The results of Treg cells in the pathogenesis of RA are still inconsistent. Van Amelsfort et al found that the proportion of Treg cells in peripheral blood and synovial fluid of patients with RA increased, and the synovial fluid was higher than peripheral blood. Miyara et al [19] reported that there were a large number of Tregs in RA inflammatory joint tissues. Walter et al [20] reported that the changes of peripheral blood Treg cells in patients with chronic RA were not statistically significant compared with the control group. Flores-Borja $\mathrm{F}$ et al [21-24] found that the number of CD24hiCD38hi Bregs in peripheral blood of patients with RA was significantly reduced, and could not inhibit Th17 response, nor could it promote the differentiation of CD4+T cells into Treg. The number of patients with Bregs was negatively correlated with the degree of disease activity. CD24hi CD27+B cells, IL-10+TIM1+B cells, and IL-10+CD5+CD1dhi B cells were significantly reduced in peripheral blood of patients with RA. However, Kim et al [25] found that the number of IL-10+Bregs in peripheral blood of patients with RA increased. Kawashiri et al [26] found that the proportion of Treg cells in active RA patients was lower than that in the control group, but there was no difference between the remission phase and the control group, and the Treg cell ratio was negatively correlated with the DAS28 score. Patients with RA not only had a reduced number of Treg cells, but also showed defects in immunosuppressive function. Ehrenstein et al believed that the number of Treg cells might be negatively correlated with the severity of RA. Treatment of RA with monoclonal antibody could increase the number of Treg cells in peripheral blood of patients. There was no significant difference in peripheral blood CD4+CD25+iTreg cells between the RA patients and the control group, but the synovial fluid CD4+CD25+Treg cells were significantly increased [27]. Astry et al [28] reported that Foxp 3 was expressed at low levels in peripheral blood of patients with RA, while IL-17 was significantly higher than the control group, leading to inflammation and bone destruction. Boissier et al [29] found that the number and function of Treg in peripheral blood of patients with RA were insufficient, and the treatment with TNF- $\alpha$ antagonists could significantly increase the number of Treg. Thiolat et al [30] evaluated the role of the Treg-specific secretory cytokine IL-35 in a mouse model of RA by non-viral gene transfer, and found that the clinical score of arthritis induced by IL-35 gene transfer was higher than that of empty vector gene transfer induced arthritis. The clinical score was significantly increased. Considering the controversy over the value of Treg cells in RA patients, Wang et al [31] observed changes in Th17/Treg ratio and found that RA, especially in active patients, had an increased Th17/Treg ratio, while healthy people had a lower Th17/Treg ratio. The above findings were inconsistent and might be due to different stimuli that induce IL-10 secretion in vitro. Tumor necrosis factor alpha (TNF- $\alpha$ ) in RA patients can inhibit the function of Treg cells by inducing Foxp3 phosphorylation [32], which was the main factor leading to the impaired function of Treg cells, which made Treg cells lose their inhibitory function. Therefore, in RA, Treg cells were not completely effective in inhibiting the inflammatory response. Although the proliferation and differentiation of TGF- $\beta$ in Tregs and Th17 have been well established, the role of TGF- $\beta$ in the differentiation of IL-17+Foxp $3+$ T cells has not been agreed [33-35]. Most believe that Tregs produce IL-17 dependent on TGF- $\beta$ [34]. TGF- $\beta$ inhibitors can reduce IL-17 production, but can not be eliminated. In the presence of antigen presenting cells, TGF- $\beta$ and IL-2 maximize the level of IL-17+Foxp3+T cells. In mice with Treg cells missing, symptoms such as arthritis were significantly aggravated. Adoptive transfer of CD4+CD25+Treg cells to CIA mice can cure RA. Most experiments have shown that Treg is involved in the progression of RA, which induces Treg expansion in vitro, enhances Foxp 3 gene expression, promotes Treg efficacy, enhances its immunosuppressiveness, and opens up new ideas for the treatment of RA.

\subsection{Th17/ Treg Imbalance and Systemic Lupus Erythematosus}

\subsubsection{Th17 Cell and Systemic Lupus Erythematosus}

Systemic lupus erythematosus (SLE) is a non-organ-specific autoimmune connective tissue disease. Most patients have typical butterfly or discoid erythema on the cheek, It often involved in the heart, kidneys and other organs. Lupus nephritis (LN) is one of the most serious clinical manifestations of SLE. The pathogenesis of SLE is caused by genetic, environmental, estrogen, infection, drugs and other factors, but the specific pathogenesis is still not very clear. Animal experiments have shown that the number of Th17 cells is significantly increased in the SLE mouse model, and high levels of serum IL-17A are associated with renal immune complex deposition and complement activation in the SLE mouse model [36, 37]. Araújo et al [38, 39] reported that IL-17A expression levels and Th17 cells were significantly increased in SLE patients, IL-17A mRNA in activated peripheral blood mononuclear cells (PBMCs) and IL-17 CD4+ production. The expression of CCR4 and CCR6 receptors in $\mathrm{T}$ cells was significantly increased. The IL-4-producing CD4+T cells and serum/plasma IL-17 and IL-17A concentrations were positively correlated with the SLE disease activity index (SLEDAI) [40]. The expression levels of cytokines such as IL-17, IL-23 and IL-27 mRNA 
associated with Th17 cells in urine sediment were significantly increased, and IL-17 and ROR $\gamma \mathrm{t}$ expression were up-regulated. The expression levels of IL-17A and IL-23 in glomeruli of LN patients were positively correlated with SLEDAI and histological activity index [41]. The expression level of IL-17 in renal biopsy tissues was closely related to clinical parameters such as hematuria, urine protein and blood urea nitrogen. It indicated that the number of Th17 cells was closely related to the severity of SLE disease, and Th17 cells and IL-17A played an important role in the pathogenesis of SLE.

\subsubsection{Treg Cell and Systemic Lupus Erythematosus}

The results of Treg cells in the pathogenesis of SLE are controversial. In most cases, Treg cells in peripheral blood of SLE patients are significantly reduced, and some people believe that Treg cells are reduced or functionally impaired. Chowdary found that co-culture of Treg cells with autologous effector T cells in SLE patients can attenuate the inhibitory function of Treg cells, while the inhibitory function of effector $\mathrm{T}$ cells co-cultured with normal people was not affected, suggesting that the effector $\mathrm{T}$ cells in peripheral blood of SLE patients may have resistance to the inhibition of Treg cells. There were also researches [42] found that the proportion of CD25-T cells and CD25-FoxP3+cells in SLE patients increased, and in vitro experiments confirmed that these cells were different from conventional Treg cells, and could not effectively suppress effector T cells. Humrich et al [43] found that early SLE, IL-2 reduction can promote the activation of initial $\mathrm{T}$ cells, destroy the balance of Treg and effector $\mathrm{T}$ cells, and accelerate the progression of the disease. Lee et al [44] reported that $C D+4 C D+25$ Treg cells in peripheral blood of patients with SLE were lower than those in the control group, and their inhibitory function was also reduced. Gomez et al [45] believed that Treg had nothing to do with the pathogenesis of SLE. Its number and function were not significantly different from the control group, and for the inhibition of Treg cells, self-effector $\mathrm{T}$ cells were tolerated. Differences in findings may be related to different stages of SLE disease, drug effects, or ethnic differences. Jia et al [46] reported that Th17 cells, Th17/Treg and IL-6, IL-17, IL-23 were higher in the active SLE patients than in the inactive phase and the control group, and Treg cells and TGF- $\beta$ were lower than the inactive phase. In the control group, the non-active patients had higher Th17 cells, Th17/Treg and IL-6, IL-17 and IL-23 than the control group, and Treg cells were lower than the control group. It indicated that Th17 cells and Treg cells and related cytokines were involved in the pathogenesis of SLE, and their growth and decline were related to SLE activity. It was suggested that the imbalance of Th17/Treg played an important role in the occurrence and development of SLE.

\subsection{Th17 Cells and Psoriasis}

Psoriasis, commonly being known as Psoriasis (Ps), is a chronic immune disorder skin disease characterized by keratinocyte proliferation and inflammatory cell infiltration.
It often has red, scaly or white patches of skin, dry, itchy or cracked and other symptoms. Diani et al [47] found that Th1 and Th17-associated cytokines were overexpressed in skin lesions and plasma of patients with psoriasis, which showed an increase in IL-23 and Th17 cells producing IL-17A. Transfer of the IL-17A gene into the CIA model revealed that the IL-17A gene can induce the expansion of IL-17RA(+)CD11b(+)Gr1(low) osteoclast precursors [48, 49]. The number of IL-17+CD4+T cells, IL-22+ CD4+T cells and IL-17 and IL-22 secreted by PBMCs in PBMCs of Ps and PsA patients were significantly increased [50]. The number of Th17 cells and IL-17A expression in synovial tissue and synovial fluid of PsA patients increased significantly, and the level of IL-17A was significantly correlated with the degree of joint damage. The level of Th17 marker transcription factor RORc and the levels of inflammatory factors IL-1 $\beta$, IL-6, IL-23 were significantly increased. The number of Th17 cells was significantly increased, and it was related to the degree of disease activity, from asymptomatic to skin lesions. The $\mathrm{CD} 3+, \mathrm{CD} 4+$, and Foxp3+ cells gradually increased, and the ratio of Treg/CD4+T cells in the lesion site was much higher than that in the non-lesional site. The occurrence of Ps and the degree of lesions were associated with decreased levels of Foxp 3 mRNA. Foxp3+Treg cells were reduced in advanced lesions, and Treg cells were significantly elevated in treatment with effective clinical symptoms [51]. The ratio of Th17, Foxp3+Treg cells and Th17/Treg cells increased significantly in children with seizures, and was positively correlated with the degree of inflammation [52]. Foxp3+Treg cells were reduced in the lesions of advanced patients, and IL-17A+/Foxp 3+/CD4 + triple positive cells were present in the lesions of severe patients, suggesting that the severity of the disease was negatively correlated with the number of Foxp3+Treg cells. After receiving infliximab in patients with Ps, the number of Treg cells was significantly increased and the clinical symptoms were significantly improved [51]. As a commonly used drug for the treatment of Ps, vitamin D3 can induce dendritic cells (DCs) in the epidermis and dermis to differentiate into different types of Treg cells, maintain immune tolerance, and relieve clinical symptoms [53]. Therefore, Th17/Treg cell imbalance plays an important role in the pathogenesis of Ps.

\subsection{Th17/Treg Imbalance and Multiple Sclerosis}

Multiple sclerosis (MS) is a chronic progressive central nervous system demyelinating inflammatory autoimmune disease caused by immune tolerance disorder. It mainly caused by reactivity CD4+T cells in myelin antigen 2478 itself immune response. Reactive $\mathrm{CD} 8+\mathrm{T}$ cells and $\mathrm{B}$ cells are involved in the immune damage process. Experimental autoimmune encephalomyelitis (EAE) in mice found that Th17 cells were important pathogenic factors, and the blockade of Treg cell function was positively correlated with the degree of disease progression. Correale et al [54] found that there were inhibitory B cells in MS patients, and the isolated B cells can inhibit the proliferation of T cells and the 
production of IFN- $\gamma$. The clinical prognosis of MS patients infected with helminths was better. It is speculated that the increase of Bregs secreting il-10 may improve the condition of MS patients infected by worms. Brain biopsy of MS patients revealed that IL-17A gene was overexpressed and Th17 cells were significantly increased. The number of Th17 cells in the central nervous system of MS patients was significantly increased, and Th17 cells can also weaken the blood-brain barrier and promote the entry of other immune cells into the central nervous system. Th17 cells were more likely to pass the blood-brain barrier than Th1 cells, and Treg cells were reduced and functionally impaired in MS patients. Knippenberg S et al [55] found. Bregs cells secreting IL-10 in peripheral blood of patients with relapsing remitting multiple sclerosis (RRMS) were significantly lower than those in control and remission MS patients. Kleinewietfeld et al [2] reported that the number and/or function of Treg in MS patients was abnormal, and the Foxp3 signaling and protein expression of Treg cells in RRMS patients were abnormal, leading to a decrease in Treg cell function and promoting disease. Schubert et al [56] found that after treatment with IFN- $\beta$, regulatory $\mathrm{CD} 19+\mathrm{CD} 24++\mathrm{CD} 38++$ transitional $\mathrm{B}$ cells and IL-10 were significantly increased in peripheral blood of patients with RRMS. After blocking the signal transduction pathway of heparin-binding cytokines in patients, Treg cells were significantly increased, EAE symptoms were significantly improved, and there was no adverse reaction. Therefore, considering treatment options from the perspective of Th17 and Treg cells can provide a new direction for MS disease. After Grützke et al [57] treatment with fingolimod, the number of CD38+CD27-CD24+CD5+ Breg cells in peripheral blood increased significantly, and the function of IL-10 secretion was also significantly enhanced. Studies have shown that Th17/Treg imbalance plays an important role in the pathogenesis of MS, and its changes can be used to judge the condition, observe the effect and monitor the prognosis.

\subsection{Th17/Treg Imbalance and Inflammatory Bowel Disease}

Inflammatory bowel disease (IBD) is a non-specific colonic inflammation, including Crohn's disease (CD) and ulcerative colitis (UC). It is a kind of intestinal inflammatory disease whose etiology and pathogenesis are not very clear. The study believes that genetic, environmental, microbial and immune factors play a role in the occurrence and development of $\mathrm{CD}$, of which immune factors play a key role [58]. Studies have shown that Th17 and Treg cells play an important role in the development of CD intestinal inflammation [59]. Mouse enteritis models and clinical studies have found that IL-23 is the core virulence factor in the development of spontaneous enteritis and infection-induced enteritis, and IL-23 receptor gene variants can reduce the severity of IBD disease. IL-17 is expressed in the small intestine inflammation site of IBD, and the accumulation of Th17 cells exists in the lamina propria, and antigen-presenting cells (APC) can efficiently induce Th17 cells growing through IL-1 $\beta$, IL-6 and IL-23.
IL-17+IFN $\gamma+\mathrm{T}$ cells and IL-17+Foxp $3+\mathrm{T}$ cells are present in the colonic mucosa. The role of Th17 in IBD remains controversial. Some believe that Th17 cells can mediate intestinal inflammation by releasing inflammatory factors, while others believe that Th17 cells have a protective effect on intestinal mucosa. Chao et al [60] believed that Th17 cells can mediate chronic inflammation and autoimmune diseases, and have a relationship with Treg cells. Maul et al found that CD4+CD25(hi) Fox-p3+T cells with high expression during IBD activity were lower than inactive. that the proportion of Treg cells in peripheral blood of patients with active IBD decreased, and the remission period increased, suggesting that Treg cells had immunoinflammatory protective effects in the formation of intestinal inflammation in patients with IBD. Th17/Treg cell balance was an important factor in maintaining intestinal immune homeostasis, and its imbalance may be one of the causes of human IBD. CD animal models have demonstrated that IL-23R expressed by $\mathrm{T}$ cells was essential for initial $\mathrm{T}$ cell proliferation and differentiation into IL-17A+IFN- $\gamma+$ pathogenic T cells, and IL-17A+IFN- $\gamma+$ T cells promoted intestinal tract The development of inflammation, defects in the IL-23/Th17 pathway, can improve the condition of animals [61]. IL-17A also had pro-inflammatory and anti-inflammatory effects in $\mathrm{CD}$ animal models, suggesting that the role of IL-17A in CD was very complex [62]. Jiang et al [63] reported that the expression levels of other effector cytokines (such as IL-21 and IL-22) protein and mRNA in IL-17A, IL-17F, I L-23 and Th17 cells were significantly increased in active CD enteritis tissues. Treg cells in UC patients were also susceptible to polarization to IL-17-secreting cells, and IL-17+Foxp3+T cells inhibited T cell activation and stimulate inflammation in the colonic mucosa [64]. It was suggested that the imbalance of Th17/Treg was related to the progression of inflammatory bowel disease. Zhao et al [65] found the proportion of peripheral blood Treg cells in patients with mild to moderate active $\mathrm{CD}$ was significantly lower than that in the remission phase and the control group. The proportion of Th17 cells was higher than that in the remission phase, and the proportion of Th17/Treg cells was abnormally changed and the inflammatory index reflecting the $\mathrm{CD}$ disease activity was ESR and C. The level of reactive protein was negatively correlated, suggesting that Th17/Treg cell imbalance was involved in the development and progression of CD intestinal inflammation.

\subsection{Th17/Treg Imbalance and Primary Sjogren's Syndrome}

Primary sjogren's syndrome (PSS) is a chronic inflammatory autoimmune disease mainly invading the exocrine glands such as salivary glands and lacrimal glands. It is one of the most common diffuse connective tissue diseases in China, which is characteristic of lymphocyte infiltration. The common symptoms are obvious dry eyes, and other system damage, which seriously affects the quality of life of patients. The pathogenesis of SS is complex. It may be some factors such as heredity, environment, infection and abnormal sex hormones acting together on the body, leading to tissue damage caused 
by infiltration of inflammatory mediators, abnormal cellular immune function, and then developed into SS. Sudzius et al [66] believed that the imbalance of T cells in peripheral blood was an important factor in the pathogenesis of SS. Wang et al [67] found that there were a lot of lymphocyte infiltration and IL-17 cell expression in the labial gland of SS patients, while the control group had no lymphocyte infiltration and IL-17 positive expression, suggesting that IL-17 was involved in the process of SS inflammatory injury. Yu et al [68] reported that the expression of IL-17, IL- 6 and TNF- $\alpha$ in the labial gland of PSS patients was significantly higher than that in the control group, suggesting that Th17 cells and their secreted cytokines play a important role in the pathogenesis and development of SS. Wang et al [69] found that Th17 cells in SS patients were significantly higher than the control group, and high Th17 expression was associated with the pathogenesis of SS. Sumida et al [70] studied the expression of Th17 cells in the animal model of M3R-/- induced salivary gland dry mice, and found that Th17 cells and IL-17 were detected in the salivary glands and serum of mice, indicating IL-17 in SS model mice rised. The level of IL-17 in the labial gland and peripheral blood of C57BL/6. NOD-Aec1Aec2 SS mouse model showed that IL-17 expression was highest at 4 weeks in SS mice, and IL-17 expression level gradually decreased with mouse growth, and IL-17 was not found in the labial gland and peripheral blood of the normal C57BL/6J mouse model. The results were similar to the expression of IL-17 in the labial gland and peripheral blood of human SS patients, confirming that the high expression of Th17 cells was associated with the pathogenesis of SS. Zhu et al [71] reported that 60 cases of SS patients with lip gland tissue and serum IL-17 levels were significantly higher than 30 normal controls. The expression of Foxp3m RNA in the labial gland tissue decreased. Alunno et al [72] found that the number of Treg in peripheral blood of patients with PSS was lower than that of the normal control group, and the expression of $\mathrm{CD}+4 \mathrm{CD}+25$ low $\mathrm{T}$ cells in the labial gland was significantly higher than that in peripheral blood. Inferring the accumulation of Treg in the target organ inflammation region, which was related to the inhibition of autoreactive $\mathrm{T}$ cells and regulation of local immune response, may be used as a reverse regulation or negative feedback mechanism [73]. Zheng et al [74] found that peripheral blood CD4+CD25+high regulatory $\mathrm{T}$ cells were significantly lower in SS patients than in healthy subjects, suggesting that the expression of $\mathrm{CD} 4+\mathrm{CD} 25+$ high regulatory $\mathrm{T}$ cells in peripheral blood of SS patients was related to the pathogenesis of SS. Li et al [75] reported that CD4+CD25+Foxp3+ cells in SS patients were significantly lower than the control group, indicating that the decrease in the number of Treg cells in peripheral blood may be involved in the pathogenesis of SS. The above results indicated that Th17/Treg immune imbalance is involved in the development of SS.

\subsection{Th17/Treg Disorders and Autoimmune Thyroid Diseases}

Autoimmune thyroid diseases (AITD) are the most common thyroid diseases except iodine deficiency disorders, and are also common organ-specific autoimmune diseases in humans. AITD mainly includes diffuse toxic goiter (Graves disease) and Hashimoto thyroiditis (HT), characterized by hyperthyroidism and hypothyroidism. The common characteristic is the deficiency of immune tolerance of thyroid tissues, which leads to multiple antibody positive and lymphocyte infiltration. AITD has a complex etiology and has a similar genetic and immunological basis. Exposure to environmental causes for a prolonged period of time can promote disease in people with specific genetic backgrounds. In the past, the correlations between AITD and Th1/Th2 subpopulations and cytokines were extensively studied. It was believed that the pathogenesis of AITD was closely related to Th1 and Th2. With the deepening of research, it has found that the traditional Th1 and Th2 cell imbalances can still not well explain the pathogenesis of AITD. Glick et al [76] found no significant difference in the number of Treg CD4+T cells in peripheral blood of AITD patients, but the inhibition ability was lower than that of the control group. Marazuel et al [77] reported that the number of Treg cells in peripheral blood of AITD patients was significantly higher than that of the control group, and its inhibitory function was significantly decreased. Although the results of Treg cell numbers in AITD patients were inconsistent, the decline in the function of Treg cells has been determined, which suggested that the number of Treg cells changed or decreased in function to participate in the development of AITD. Wang et al [78] first simulated the human GD model with female rhesus monkeys and found that the number of Treg cells decreased and the ratio of Th17 cells/Treg increased. Kahaly et al [79] found that the immune suppression function of Treg cells in peripheral blood of patients with GD was significantly lower than that of the control group, but the number of Treg cells was not significantly different from that of the control group, which suggested that the decreased function of Treg cells was closely related to the pathogenesis of GD. Tang et al [80] detection of peripheral blood CD4+ and CD25 in patients with GD+Treg cells also gave the same results. Peng et al [81] found that the number of Th17 cells in peripheral blood of patients with newly diagnosed GD was higher than that of the control group. Mao et al [82] reported that the number of CD4+CD25+Foxp3+Treg in peripheral blood of patients with newly diagnosed GD was significantly decreased, and negatively correlated with TSHR antibody titer. Klatka et al [83] found that the percentage and absolute value of peripheral blood Treg increased in adolescent GD patients treated with methimazole. These studies suggest that changes in the number and dysfunction of Treg are associated with the onset and prognosis of GD. Chen [84] found that the expression levels of IL-17A mRNA and serum IL-17 in thyroid tissue of patients with HT were significantly higher than those of the control group, and the HT-low group was higher than the HT-normal group, while Foxp3 mRNA expression and IL-10 level were significantly lower than the control group, but there was no significant difference between the HT-A low group and the HT-induced normal group. It was suggested that the immune suppressive function 
of Treg cells in thyroid tissue and peripheral blood of HT patients decreased, while the Th17 cell response was enhanced. Th17/Treg immune imbalance may be involved in the occurrence and development of HT. Zhao [85] found that patients with Graves disease and HT had elevated Th17/CD4+T cells in peripheral blood, especially the latter was more obvious. Therefore, it can be used as one of the diagnostic basis of AITD, especially for the diagnosis of HT. There was a significant positive correlation between Th17/CD4+ $\mathrm{T}$ cells and $\mathrm{Tg}-\mathrm{Ab}$ in $\mathrm{HT}$ patients, but no significant correlation with TPO-Ab. There was no significant correlation between Th17/CD4+ T cells and thyroid autoantibodies in patients with Graves disease. Short-term treatment with levothyroxine tablets and methimazole failed to alter the number of Th17/CD4+ T cells and thyroid autoantibodies in peripheral blood of patients with hypothyroidism in HT and Graves. All in all, Th17/Treg cell dysregulation was involved in the development of AITD and played an important role in the pathogenesis of HT and Graves disease.

\subsection{Th17/ Treg Imbalance and Autoimmune Hepatitis}

Autoimmune liver disease is an autoimmune disease in which the liver is the target organ. The pathogenesis is related to the immune regulation disorder of the body. Autoimmune hepatitis (AIH), which is mainly caused by hepatocyte injury, is the most common. Although genetic and environmental factors play an important role in the pathogenesis of $\mathrm{AIH}$, the exact mechanism remains unclear [86]. Zhao et al [87] reported that IL-17+lymphocyte infiltration (mainly CD4+ phenotype) was significantly increased in patients with $\mathrm{AIH}$, and the degree of infiltration was positively correlated with the degree of inflammation and abnormality of AIH, and Th17 cells and serum IL-17 were significantly higher than the control group, which indicated that Th17 cell proliferation played a key role in the pathogenesis of AIH. Ferri et al [88] found that AIH patients not only reduced the number of Treg cells, but also impaired function. Longhi et al reported that Treg cells in AIH patients can be expanded and reconstituted (CD4+CD25-cells), and their immunosuppressive function and Foxp3 expression levels were higher than freshly isolated Treg cells. It was confirmed that CD4+CD25-containing Treg cells produced in AIH patients contained more IL-17+RORC+cell populations, and these cells were less effective in inhibiting CD25-cell proliferation than Treg cells from CD4+CD25+cells. Holder et al [89] reported that the number of CD4+CD25+Treg cells was decreased and function was lost in AIH patients, and IL-17 was highly expressed in hepatic inflammatory cell infiltration. Peiseler et al [90] found that the ratio of peripheral blood transcription factors ROR $\gamma \mathrm{t}$ and Foxp3 in AIH patients was positively correlated with the degree of liver lesions, and the treatment of Treg cells was significantly increased. Therefore, it is speculated that Th17/Treg imbalance is closely related to the occurrence and development of AIH.

\section{Conclusion}

Autoimmune diseases (AIDs) are polygenic hereditary diseases caused by interactions among immune, environmental and genetic factors. Th17 cells induce inflammatory responses mainly by secreting various cytokines such as IL-17, IL-6 and TNF- $\alpha$. Treg cells inhibit autoreactive T cells through a variety of mechanisms, maintain immune tolerance, and limit immune damage caused by excessive immune responses. At present, research on Th17/Treg cells has been widely carried out on a global scale, and has become a research hotspot especially in AID. The results show that: (1) Th17/Treg cells are involved in the AID development of rheumatoid arthritis, systemic lupus erythematosus, psoriasis, multiple sclerosis, inflammatory bowel disease, primary dryness syndrome, autoimmune hepatitis and autoimmune thyroid disease, which provides an experimental basis for the diagnosis of the disease; (2) Dynamic monitoring of changes in Th17/Treg during the disease process is conducive to observing the condition, evaluating the efficacy, and judging the prognosis; (3) the regulation of il-17 or il-17R, blocking the inflammatory effect of il-17, effectively amplifying Treg in vitro, adoptive infusion of Treg in patients with AID, or induction of CD4+ CD25-treg into CD4+CD25+ Treg in vivo, provide possible targets for the treatment of AID and opening up a new approach for the treatment of AID; (4) Combined with the basic theory of immunology, through the intervention of Th17/Treg ratio, inhibiting excessive hyperthyroidism of Th17 cells, or promoting the role of Treg cells, it provides a theoretical basis for effective prevention of AID. However, Th17/Treg cells are subject to the interaction and dose-dependent regulation of many cytokines and transcription factors, as well as the interaction and influence of multiple signal transduction pathways, and influenced by the number of samples, race, region and different stages of disease, so the results of Th17/Treg cells in AID are not the same. Therefore, further in-depth study of Th17/Treg cells in a large sample of AID, or Metal analysis of relevant data, will contribution to the diagnosis of disease accuracy, refined treatment, precision prevention, and important guiding significance of precise regulation of drug targets, and the meticulous analysis of the pathogenesis, which can also provide the reliable experimental data and theoretical basis.

\section{Acknowledgements}

Cangzhou Key R \& D Plan and Guidance Project (Project No.: 1833020011).

\section{References}

[1] Shabgah AG, Fattahi E, Shahneh FZ. Interleukin-17 in human inflammatory diseases. Postepy Dermatol Alergol, 2014, vol. 31, pp. 256-261.

[2] Kleinewietfeld $M$, Hafler DA. Regulatory $T$ cells in autoimmune neuroinflammation [J]. Immunol Rev. 2014, vol. 259, pp. 231-244. 
[3] Ma A, Yang Y, Wang Q, et al. Anti-inflammatory effects of oxymatrine on rheumatoid arthritis in rats via regulating the imb-alance between Treg and Th17 cells [J]. Mol Med Rep. 2017, vol. 15, pp. 3615-3622.

[4] Hou X, Song J, Su J, et al. CD4(+)Foxp3(+) Tregs protect against innate immune cell-mediated fulminant hepatitis in mice [J]. Mol Immunol. 2015, vol. 63, pp. 420-427.

[5] Miossec P, Kolls JK. Targeting IL-17 and TH17 cells in chronic inflammation [J]. Nat Rev Drug Discov, 2012, vol. 11, pp. 763-776.

[6] Ghoreschi K, Laurence A, Yang XP, et al. Generation of pathogenic $\mathrm{T}(\mathrm{H}) 17$ cells in the absence of TGF- $\beta$ signalling $[\mathrm{J}]$. Nature, 2010, vol. 467, pp. 967-971.

[7] Annunziato F, Cosmi L, Liotta F, et al. Type $17 \mathrm{~T}$ helper cells-origins, features and possible roles in rheumatic disease [J]. Nat Rev Rheumatol, 2009, vol. 5, pp. 325-331.

[8] Mangodt TC, Van Herck MA, Nullens S, et al. The role of Th17 and Treg responses in the pathogenesis of RSV infection [J]. Pediatr Res, 2015, vol. 78, pp. 483-491.

[9] Ballke C, Gran E, Baekkevold ES, et al. Characterization of Regulatory T-Cell Markers in CD4+ T Cells of the Upper Airway Mucosa [J]. PLoSOne, 2016, vol. 11, pp. e0148826

[10] Abd Al Samid M, Chaudhary B, Khaled YS, et al. Combining FoxP3 and Helios with GARP/LAP markers can identify expan-ded Treg subsets in cancer patients [J]. Oncotarget, 2016, vol. 7, pp. 14083-14094.

[11] Pandiyan P, Zhu JF. Origin and functions of pro-inflammatory cytokine producing Foxp3(+) regulatory T cells [J]. Cytokine, 2015, vol. 76, pp. 13-24.

[12] Hall BM, Verma ND, Tran GT, et al. Distinct regulatory $\mathrm{CD} 4+\mathrm{T}$ cell subsets; differences between naïve and antigen specific T regulatory cells [J]. Curr Opin Immunol, 2011, vol. 23, pp. 641-647.

[13] Kugyelka R, Kohl Z, Olasz K, et al. Enigma of IL-17 and Th17 cells in rheumatoid arthritis and in autoimmune animal mod-els ofarthritis [J]. Mediators Inflammation, 2016, pp. 6145810

[14] van Hamburg JP, Asmawidjaja PS, Davelaar N, et al. Th17 cells, but not Th1 cells, from patients with early rheumatoid arthri-tis are potent inducers of matrix metalloproteinases and proin amatory cytokines upon synovial fbroblast interaction, including autocrine interleukin-17A production [J]. Arthritis Rheumatism, 2011, vol. 63, pp. 73-83.

[15] Wang $T$, Sun $X$, Zhao J, et al. Regulatory $T$ cells in rheumatoid arthritis showed increased plasticity toward Th17 but retained suppressive function in peripheral blood [J]. Ann Rheum Dis, 2015, vol. 74, pp. 1293-1301.

[16] Lee YH, Bae SC. Associations between circulating IL-17 levels and rheumatoid arthritis and between IL-17 gene polymor-phisms and disease susceptibility: a meta-analysis [J]. Postgrad Med J, 2017, vol. 93, pp. 465-471.

[17] Wei M. Efficacy and safety of monoclonal antibodies targeting interleukin-17 pathway for inflammatory arthritis: a meta-analysis of randomized controlled clinical trials [J]. Drug Des Devel Ther, 2016, vol. 10, pp. 2771-2777.

[18] Attridge K, Wang CJ, Wardzinski L, et al. IL-21 inhibits T cell
IL-2 production and impairs Treg homeostasis [J]. Blood, 2012, vol. 119, pp. 4656-4664.

[19] Miyara M, Gorochov G, Ehrenstein M, et al. Human FoxP3 regulatory $\mathrm{T}$ cells in systemic autoimmune diseases $[\mathrm{J}]$. Autoimm-un Rev, 2011, vol. 10, pp. 744-755.

[20] Walter GJ, Fleskens V, Frederiksen KS, et al. Phenotypic, Functional, and Gene Expression Profiling of Peripheral CD45RA+and CD45RO+CD4+CD25+CD127 (low) Treg Cells in Patients With Chronic Rheumatoid Arthritis [J]. Arthritis Rheumatol, 2016, vol. 68, pp. 103-116.

[21] Flores-Borja F, Bosma A, Ng D, Reddy V, et al. CD19+CD24hiCD38hiB cells maintain regulatory $\mathrm{T}$ cells while limiting TH1 and TH17 differentiation [J]. Sci Transl Med, 2013, 5 (173): 2013, vol. 5, pp. 173ra23.

[22] Ma L, Liu B, Jiang Z, et al. Reduced numbers of regulatory B cells are negatively correlated with disease activity in patients with new-onset rheumatoid arthritis [J]. Clin Rheumatol, 2014, vol. 33, pp. 187-195.

[23] Daien CI, Gailhac S, Mura T, et al. Regulatory B10 cells are decreased in patients with rheumatoid arthritis and are inversely correlated with disease activity [J]. Arthritis Rheumatol, 2014, vol. 66, pp. 2037-2046.

[24] Cui D, Zhang L, Chen J, et al. Changes in regulatory B cells and their relationship with rheumatoid arthritis disease activity [J]. Clin Exp Med, 2015, vol. 15, pp. 285-292.

[25] Kim J, Lee HJ, Yoo IS, et al. Regulatory B cells are inversely associated with disease activity in rheumatoid arthritis [J]. Yon-sei Med J, 2014, vol. 55, pp. 1354-1358.

[26] Kawashiri SY, Kawakami A, Okada A, et al. CD4+CD25 (high) CD127 (low/-) Treg cell frequency from peripheral blood cor-relates with disease activity in patients with rheumatoid arthritis $[\mathrm{J}]$. J Rheumatol, 2011, vol. 38 pp. $2517-2521$

[27] Möttönen M, Heikkinen J, Mustonen L, et al. CD4+CD25+T cell with the phenotypic and functional characteristics of regul-atory $\mathrm{T}$ cells are enriched in the synovial fluid of patientswith rheumatoid arthritis [J]. Clin Exp Immunol, 2005, vol. 140 pp. $360-367$.

[28] Astry B, Venkatesha SH, Moudgil KD. Involvement of the IL-23/IL-17 axis and the Th17/Treg balance in the pathogenesisand control of autoimmune arthritis $[\mathrm{J}]$. Cytokine. 2015, vol. 74. pp. 54-61.

[29] Boissier MC, Assier E, Biton J, et al. Regulatory T cells (Treg) in rheumatoid arthritis [J]. Joint Bone Spine, 2009, vol. 76. pp. $10-14$.

[30] Thiolat A, Denys A, Petit M, et al. Interleukin-35 gene therapy exacerbates experimental rheumatoid arthritis in mice [J]. Cytokine, 2014, vol. 69. pp. 87-93.

[31] Wang W, Shao S, Jiao Z, et al. The Th17/Treg imbalance and cytokine environment in peripheral blood of patients with rheumatoid arthritis [J]. Rheumatol Int, 2012, vol. 32. pp. 887-893.

[32] Nie H, Zheng Y, Li R, et al. Phosphorylation of FOXP3 controls regulatory $\mathrm{T}$ cell function and is inhibited by TNF- $\alpha$ in rheumatoid arthritis [J]. Nat Med, 2013, vol. 19. pp. 322-328. 
[33] Liang H, Yi L, Wang X, et al. Interleukin-17 facilitates the immune suppressor capacity of high- grade glioma-derived CD4(+)CD25(+)Foxp3(+) T cells via releasing transforming growth factor beta [J]. Scand J Immunol, 2014, vol. 80. pp. $144-150$.

[34] Wang X, Wang $\mathrm{W}, \mathrm{Xu}$ J, et al. All-trans retinoid acid promotes allogeneic corneal graft survival in mice by regulating Treg-Th17 balance in the presence of TGF- $\beta[\mathrm{J}]$. BMC Immunol, 2015, vol. 16. pp. 17.

[35] Hovhannisyan Z, Treatman J, Littman DR, et al. Characterization of interleukin-17-producing regulatory $\mathrm{T}$ cells in inflamed intestinal mucosa from patients with inflammatory bowel diseases [J]. Gastroenterology, 2011, vol. 140. pp. $957-965$.

[36] Kang HK, Liu M, Datta SK. Low-dose peptide tolerance therapy of lupus generates plasmacytoid dendritic cells that cause expansion of autoantigen-specific regulatory $\mathrm{T}$ cells and contraction of inflammatory Th17 cells [J]. J Immunol, 2007, vol. 178. pp. 7849-7858.

[37] Hsu HC, Yang PA, Wang J, et al. Interleukin 17-producing T helper cells and interleukin 17 orchestrate autoreactive germinal center development in autoimmune BXD2 mice [J]. Nat Immunol, 2008, vol. 9. pp. 166-175.

[38] Araújo JA, Mesquita D, de Melo Cruvinel W, et al. Th17 cells and $\mathrm{CD} 4(+)$ multifunctional $\mathrm{T}$ cells in patients with systemic lupus erythematosus [J]. Rev Bras Reumatol, 2016, vol. 74. pp. 28-36.

[39] An N, Chen Y, Wang C, et al. Chloroquine autophagic inhibition rebalances Th17/Treg-mediated immunity and ameliorates systemic lupus erythematosus [J]. Cell Physiol Biochem, 2017, vol. 44. pp. 412-422.

[40] Talaat RM, Mohamed SF, Bassyouni IH, et al. Th1/Th2/Th17 /Treg cytokine imbalance in systemic lupus erythematosus (SLE) patients: Correlation with disease activity [J]. Cytokine, 2015, vol. 72. pp. 146-153.

[41] Zickert A, Amoudruz P, Sundstr m Y, et al. IL-17 and IL-23 in lupus nephritis-association to histopathology and response to treatment [J]. BMC Immunol, 2015, vol. 16. pp. 7.

[42] Von Spee-Mayer C, Siegert E, Abdirama D, et al. Low-dose interleukin-2 selectively corrects regulatory $\mathrm{T}$ cell defects in patients with systemic lupus erythematosus [J]. Ann Rheum Dis. 2016, vol. 75. pp 1407-1415.

[43] Humrich JY, Morbach H, Undeutsch R, et al. Homeostatic imbalance of regulatory and effector $\mathrm{T}$ cells due to IL-2 depriva-tion amplifies murine lupus [J]. Proc Natl Acad Sci USA, 2010, vol. 107. pp. 204-209.

[44] Lee HY, Hong YK, Yun HJ, et al. Altered frequency and migration capacity of $\mathrm{CD}+4 \mathrm{CD}+25$ regulatory $\mathrm{T}$ cells in systemic lupus erythematosus [J]. Rheumatol, 2008, vol. 47. pp. 789-794.

[45] Gomez J, Prado C, Lopez P, et al. Conserved anti-proliferative effect and poor inhibition of TNF- $\alpha$ secretion by regulatory CD4 CD25 $\mathrm{T}$ cells in patients with systemic lupus erythematosus [J]. Clin Immunol, 2009, vol. 132. pp. 385-392.

[46] Jia HY, Cheng GH. Expression and significance of Th17 cells and Treg cells and related cytokines in peripheral blood of patients with SLE [J]. Hebei Medicine, 2018, vol. 24. pp. 1277-1280.

[47] Diani MAltomare G. T helper cell subsets in clinical manifestations of psoriasis [J]. J Immunol Res, 2016, pp. 7692024.

[48] Adamopoulos IE, Suzuki E, Chao CC, et al. IL-17A gene transfer induces bone loss and epidermal hyperplasia associated with psoriatic arthritis [J]. Ann Rheum Dis, 2015, vol. 74. pp. 1284-1292.

[49] Sakkas LI, Bogdanos DP, et al. Are psoriasis and psoriatic arthritis the same disease? The IL-23/IL-17 axis data [J]. Auto-immun Rev, 2017, vol. 16. pp. 10-15.

[50] Abji F, Pollock RA, Liang K, et al. Th17 gene expression in psoriatic arthritis synovial fluid and peripheral blood compared to osteoarthritis and cutaneous psoriasis [J]. Clin Exp Rheumatol, 2018, vol. 36. pp. 486-489.

[51] Keijsers RR, Joosten I, Hendriks AG, et al. Balance of Treg versus T-effector cells during systemic treatment with adalimu-mab and topicaltreatment with calcipotriol-betamethasone dipropionate ointment. Exp Dermatol. 2015, vol. 24. pp. 65-67.

[52] Zhang L, Li Y, Yang X, et al. Characterization of Th17 and FoxP3(+)Treg Cells in Paediatric Psoriasis Patients [J]. Scand J Immunol. 2016, vol. 83. pp. 174-180.

[53] Van der Aar AM, Sibiryak DS, Bakdash G, et al. VitaminD3 targets epidermal and dermal dendritic cells for induction of distinct regulatory T cells [J]. J Allergy Clin Im munol, 2011, vol. 127. pp. 1532-1540.

[54] Correale J, Farez M, Razzitte G. Helminth infections associated with multiple sclerosis induce regulatory B cells $[\mathrm{J}]$ AnnNeurol, 2008, vol. 64. pp. 187-199.

[55] Knippenberg S, Peelen E, Smolders J, et al. Reduction in IL-10 producing B cells (Breg) in multiple sclerosis is accompanied by a reduced naïve/memory Breg ratio during a relapse but not in remission [J]. J Neuroimmunol, 2011 vol. 239. pp. 80-86.

[56] Schubert RD, $\mathrm{Hu}$ Y, Kumar G, et al. IFN- $\beta$ treatment requires $\mathrm{B}$ cells for efficacy in neuroauto immunity [J]. J Immunol, 2015, vol. 194. pp. 2110-2116.

[57] Grützke B, Hucke S, Gross CC, et al. Fingolimod treatment promotes regulatory phenotype and function of B cells $[\mathrm{J}]$. Ann Clin Transl Neurol. 2015, vol. 2. pp. 119-130.

[58] Kaser A, Zeissig S, Blumberg RS. Inflammatory bowel disease [J]. Annu Rev Immunol, 2010 vol. 28. pp. 573-621.

[59] D'Ambrosio A, Cossu A, Amendola A, et al. Lamina propria $\mathrm{CD} 4+\mathrm{LAP}+$ regulatory $\mathrm{T}$ cells are increased in active ulcerative colitis but show increased IL-17 expression and reduced suppressor activity [J]. J Crohns Colitis, 2016, vol. 10. pp. 346-353.

[60] Chao K, Zhang S, Yao J, et al. Imbalances of CD4(+)T-cell subgroups in Crohn's disease andtheir relationship with disease activity and prognosis [J]. J Gastroenterol Hepatol, 2014, vol. 29. pp. 1808-1814.

[61] Furfaro F, Gilardi D, Allocca M, et al. IL-23 Blockade for Crohn s disease: next generation of anti-cytokine therapy [J]. Exp Rev ClinImmunol, 2017 vol. 13. pp. 457-467.

[62] Wang R, Hasnain SZ, Tong H, et al. Neutralizing IL-23 is superior to blocking IL-17 in suppressing intestinal inflammation in a spontaneous murine colitis model $[\mathrm{J}]$. Inflamm Bowel Dis, 2015, vol. 21. pp. 973-984. 
[63] Jiang W, Su J, Zhang X, et al. Elevated levels of Th17 cells and Th17-related cytokines are associated with disease activity in patients with inflammatory bowel disease [J]. Inflamm Res, 2014 vol. 63. pp. 943-950.

[64] Lee SY, Lee SH, Yang EJ, et al. Metformin Ameliorates Inflammatory Bowel Disease by suppression of the STAT3 SignalingPathway and Regulation of the between Th17/Treg Balance [J]. PLoS One. 2015, vol. 10. pp. e0135858.

[65] Zhao XJ, Ma JJ, Zhu YJ, et al. Changes of Th17/Treg cell ratio and serum inflammatory activity index in patients with Crohn's disease and its clinical significance [J]. Journal of Nanjing Medical University (Natural Science), 2017 vol. 37. pp. 1000-1004.

[66] Sudzius G, Mieliauskaite D, Butrimiene I, et al. Activity of T-helper cells in patients with primary Sjogren's syndrome [J]. In Vivo, 2013, vol. 27. pp. 263-268.

[67] Wang XM. Expression of interleukin-17 in labial gland tissue and peripheral blood of patients with primary Sjogren's syn-drome [D]. Anhui Medical University, 2009.

[68] Yu ZB, Zhang FX, Zheng GM, et al. Expression and significance of Th17 cytokines in the labial glands of primary Sjögren's syndrome [J]. Journal of Clinical Medicine, 2013, vol. 28. pp. 1327-1329, 1332

[69] Wang WJ, Wen JT. Detection and significance of Th17cells in peripheral blood of patients with primary Sjogren's syndrome [J]. Chinese Journal of Laboratory Diagnosis, 2011, vol. 15. pp. 1039-1040.

[70] Sumida T, Iizuka M, Asashima H, et al. Pathogenicrole of anti-M3 Muscarinicacetylcholine receptor immune response in Sjo-gre, ns syndrome [J]. Presse Med, 2012, vol. 41. pp. 461-466.

[71] Zhu JD, Wang ZQ, Zhang PN. Observation of immune imbalance between Th17 and regulatory $\mathrm{T}$ cells in patients with Sjo-gren's syndrome [J]. Journal of Radioimmunology, 2012, vol. 25. pp. 301-303.

[72] Alunno A, Petrillo MG, Nocentini G, et al. Characterization of a new regulatory $\mathrm{CD}+4 \mathrm{~T}$ cell subset in primary Sjgren' $\mathrm{s}$ syn-drome [J]. Rheumatology (Oxford), 2013, vol. 52. pp. 1387-1396.

[73] Gao LX, Zhang FC, Ma YM, et al. Abnormal expression of regulatory $\mathrm{T}$ cells in primary Sjogren's syndrome $[\mathrm{J}]$. Chinese Journal of Rheumatology, 2012, vol. 16. pp. 477-480.

[74] Zheng HX, Yu JN, Tao YJ, et al. Expression and significance of CD4+CD25 high regulatory T cells in peripheral blood of patients with primary Sjogren's syndrome [J]. Zhejiang Journal of Practical Medicine, 2010, vol. 15. pp. 3-4.

[75] Li DM, Li XM, Li XP, et al. Detection and significance of $\mathrm{CD} 4+\mathrm{CD} 25+\mathrm{CD} 127$ low/-T cells in peripheral blood of patients with primary Sjogren's syndrome [J]. Journal of Anhui Medical University, 2009, vol. 44. pp. 482-486.

[76] Glick AB, Wodzinski A, Fu P, et al. Impairment of regulatory $\mathrm{T}$ cell function in autoimmune thyroid disease [J]. Thyroid, 2013, vol. 23. pp. 871-878.
[77] Marazuela M, García-López MA, Figueroa-Vega N, et al. Regulatory T cells in human autoimmune thyroid disease [J]. J ClinEndocrinol Metab. 2006, vol. 91. pp. 3639-3646.

[78] Wang Y, Wu LP, Fu J, et al. Hyperthyroid monkeys: a nonhuman primate model of experimental Graves' disease [J]. J Endocri-nol, 2013, vol. 219. pp. 183-193.

[79] Kahaly GJ, Shimony O, Gellman YN, et al. RegulatoryT-cells in Graves' orbitopathy: baseline findings and immunomodula-tion by anti-Tlymphocyte globulin [J]. J Clin Endo crinol Metab. 2011, vol. 96. pp. 422-429.

[80] Tang DH, Xu EL, Wu L. Quantitative and functional analysis of CD4+and CD25+regulatory T cells in peripheral blood of patients with Graves disease [J]. Journal of Navy Medicine, 2008, vol. 128. pp. 114-118.

[81] Peng D, Xu B, Wang Y, et al. A high frequency of circulating Th22 and Th17 cells in patients with new onset Graves' disease [J/OA]. PLoS One, 2013, vol. 8. pp. e68446.

[82] Mao C, Wang S, Xiao Y, et al. Impairment of regulatory capacity of $\mathrm{CD} 4+\mathrm{CD} 25+$ regulatoryTcells mediated by dendritic cell polarization and hyperthyroidism in Graves' disease [J]. J Immunol, 2011, vol. 186. pp. 4734-4743.

[83] Klatka M, Grywalska E, Partyka M, et al. Th17 and Treg cells in adolescents with Graves' disease. Impact of treatment with methimazole on these cell subsets [J]. Autoimmunity, 2014, vol. 47. pp. 201-211.

[84] Chen WZ. Study on Th17/Treg immune imbalance in patients with Hashimoto's thyroiditis [D]. Master's thesis of Nanchang University, 2012.

[85] Zhao JY. Detection and significance of Th17 cells in patients with Hashimoto's thyroiditis and Graves disease [D]. Master's thesis of Jilin University, 2012.

[86] de Boer YS, van Gerven NM, Zwiers A, et al. Genome-wide association study identifies variants associated with autoimmune hepatitis type $1[\mathrm{~J}]$. Gastroenterology, 2014, vol. 147. pp. 443-452, e5.

[87] Zhao L, Tang Y, You Z, et al. Interleukin-17 contributes to the pathogenesis of autoimmune hepatitis through inducing hepa-tic interleukin-6 expression [J]. PLoS One, 2011, vol. 6. pp. e18909.

[88] Ferri S, Longhi MS, De Molo C, et al. A multifaceted imbalance of $\mathrm{T}$ cells with regulatory function characterizes type 1 auto-immune hepatitis [J]. Hepatology, 2010, vol. 52. pp. 999-1007.

[89] Holder BS, Grant CR, Liberal R, et al. Retinoic acid stabilizes antigen-specific regulatory T-cell function in autoimmune hepatitis type 2 [J]. J Autoimmun. 2014, vol. 53. pp. 26-32.

[90] Peiseler M, Sebode M, Franke B, et al. FOXP3+regulatory T cells in autoimmune hepatitis are fully functional and not reduc-ed in frequency [J]. J Hepatol. 2012, vol. 57. pp. $125-132$. 